\title{
The angiogenic effect of dracorhodin perchlorate on human umbilical vein endothelial cells and its potential mechanism of action
}

\author{
FENG LI $^{1 *}$, TAO JIANG ${ }^{2 *}$, WEI LIU ${ }^{1}$, QUAN HU$^{1}$ and HUINAN YIN ${ }^{1}$ \\ ${ }^{1}$ Department of Burns and Plastic Surgery, The First Affiliated Hospital of the General Hospital of \\ People's Liberation Army of China, Beijing 100048; ${ }^{2}$ Department of Pharmacology, Institute of Materia Medica, \\ Chinese Academy of Medical Sciences, Beijing 100050, P.R. China
}

Received March 18, 2015; Accepted May 9, 2016

DOI: $10.3892 / \mathrm{mmr} .2016 .5442$

\begin{abstract}
Hyperglycemia is the key clinical feature of diabetes, and may induce refractory wound lesions and impaired angiogenesis. Dracorhodin perchlorate (Dra) is the major ingredient of dragon's blood and it has been used as a medicine to treat chronic wounds, such as diabetic foot, since ancient times in many cultures. The current study aimed to investigate the effect of Dra on human umbilical vein endothelial cells (HUVECs) under high-glucose (HG) stimulation and its potential mechanism. Dra was observed to increase the multiplication capacity of HUVECs both under low glucose (LG) and HG concentrations. Additionally, migration and tube formation in HUVECs was facilitated by Dra. The expression levels of Ras, mitogen-activated protein kinase (MAPK) and vascular endothelial growth factor, which are key components of the Ras/MAPK pathway, were upregulated following Dra treatment. The present study is the first report, to the best of our knowledge, of the effects of Dra on wound healing, and the association with the Ras/MAPK signaling pathway.
\end{abstract}

\section{Introduction}

Diabetes mellitus, known as simply diabetes, is a group of metabolic diseases in which there are high blood sugar levels over a prolonged period. Symptoms of high blood sugar include frequent urination, increased thirst, and increased hunger. Additionally, the increased blood glucose concentration

Correspondence to: Professor Feng Li, Department of Burns and Plastic Surgery, The First Affiliated Hospital of the General Hospital of People's Liberation Army of China, 51 Fucheng Road, Beijing 100048, P.R. China

E-mail: lifeng3737@126.com

*Contributed equally

Key words: dracorhodin perchlorate, human umbilical vein endothelial cells, angiogenesis, signaling pathway in patients with diabetes frequently results in concomitant illness including microvascular complications (nephropathy, neuropathy and retinopathy) and macrovascular complications (coronary artery disease, stroke and peripheral arterial disease) (1-3). Furthermore, high blood sugar levels are able to damage blood vessels for prolonged periods. Serious long-term complications of diabetes include heart disease, stroke, kidney failure, foot ulcers and eye damage (4-6). Patients with diabetes experience delayed wound healing, resulting in diabetes being a leading cause of non-traumatic lower extremity amputation, which is often preceded by a non-healing ulcer. It has been estimated that around $15-27 \%$ patients with diabetes require lower limb amputations, predominantly (50\%) due to infection (5).

Diabetic wounds are associated with deficient circulation and altered carbohydrate metabolism. Angiogenesis, the formation of new blood vessels from pre-existing vessels, serves a key role in the wound healing process, particularly during the proliferative phase, which involves the migration and proliferation of endothelial cells, and their differentiation into a tube-like structure. Ameliorative angiogenesis improves the wound healing process by facilitating delivery of oxygen and nutrients to the wounds whereas impaired angiogenesis has been demonstrated to be key in the impaired healing of diabetic wounds. Hence, promoting angiogenesis is a potential therapy to enhance wound healing in patients with diabetes (6).

Vascular endothelial growth factor (VEGF) is a signaling protein that stimulates vasculogenesis and angiogenesis. VEGF is an important factor in restoring the oxygen supply to tissues when blood circulation is inadequate. The normal function of VEGF is to create new blood vessels during embryonic development or bypass blocked vessels following injury (7-9). A close relationship between the reactive oxygen species induced VEGF signaling pathway and vascular disease in diabetes has been demonstrated in previous studies (10).

Herbal remedies have been widely used to accelerate the wound-healing process since ancient times, particularly in China. In recent years, the study of the use of alternative therapies and natural remedies in wound management has rapidly increased. However, their putative active compounds, molecular mechanisms of action and side effects remain to be 
fully elucidated. Dracorhodin perchlorate (Dra) is a key active ingredient isolated from the fruit of Daemonorops draco, a type of dragon's blood. Dragon's blood has long been used as a traditional Chinese medicine to improve blood circulation, prevent hemorrhages, heal wounds and as an antiseptic $(11,12)$. It has been reported that Dra is able to ameliorate the process leading to insulin resistance, enhance insulin sensitivity and inhibit high plasma lipid levels and reduce intestinal carbohydrate absorption (13). Previous studies have demonstrated a complex effect of Dra on human cells (14-18). Dra has been indicated to induce apoptosis in different cell lines (19). However, the mechanism of Dra facilitating wound healing and whether Dra may be used to relieve vessel damage in patients with diabetic ulcers remains to be fully elucidated.

The present study aimed to investigate the effect of Dra on angiogenesis and the associated signaling pathways in the human umbilical vein endothelial cells (HUVEC) under high-glucose stimulation.

\section{Materials and methods}

Cell culture and treatment. HUVECs were purchased from the Shanghai Bioleaf Biotech Co., Ltd. (Shanghai, China). HUVECs were cultured in M119 medium (Gibco; Thermo Fisher Scientific, Inc., Waltham, MA, USA) supplemented with 5\% fetal bovine serum (Gibco; Thermo Fisher Scienitific, Inc.) and $100 \mu \mathrm{g} / \mathrm{ml}$ streptomycin plus $100 \mathrm{IU} / \mathrm{ml}$ penicillin in the condition of $5 \% \mathrm{CO}_{2}$ atmosphere at $37^{\circ} \mathrm{C}$.

The experiment was designed in two groups: Low glucose concentration (LG), $5.5 \mathrm{mM}$ glucose; high glucose concentration (HG): $25 \mathrm{mM}$ glucose. For Dra treatment, $7.5 \mu \mathrm{M}$ Dra (National Institutes for Food and Drug Control, Beijing, China) was added to the medium. Following $48 \mathrm{~h}$ of culture, cells were harvested and used for experiments.

3-(4,5-dimethylthiazol-2-yl)-2,5-diphenyltetrazolium bromide (MTT) assay. HUVECs in the logarithmic phase were seeded, 5,000 cells/well in 96-well plate. Following culture in Dra and LG or HG $48 \mathrm{~h}$ at $37^{\circ} \mathrm{C}$, MTT solution $(5 \mathrm{mg} / \mathrm{ml})$ in $1 \mathrm{X} \mathrm{PBS}$ was added directly to the medium in each well, and the plate was then incubated at $37^{\circ} \mathrm{C}$ for $4 \mathrm{~h}$. The medium was then aspirated and replaced with dimethyl sulfoxide followed by the measurement of the optical density of each well at $540 \mathrm{~nm}$.

Tube formation. HUVECs were seeded into 96-well plates at 8000 cells/well on $50 \mu 1$ Matrigel (BD BioSciences, Franklin Lakes, NJ, USA). Fresh media containing Dra and LG or HG was subsequently added. Tubular structures were photographed following $6 \mathrm{~h}$. The total tube length formation was measured for quantification of angiogenesis using Image-Pro Plus software, version 6.0 (Media Cybernetics, Rockville, MD, USA).

Cell migration assay. For the migration assay, 6 well Transwell chambers (8.0 $\mu \mathrm{m}$ pores; Corning Incorporated, Corning, NY, USA) were coated with $50 \mu$ l Matrigel (BD Biosciences). A total of $10^{5}$ cells/well of HUVECs in the logarithmic phase were transferred into the upper chamber. Following $4 \mathrm{~h}$ of incubation at $37^{\circ} \mathrm{C}$, upper chambers containing Dra and $\mathrm{LG}$ or HG were set into the lower chambers. Three wells were used for each experiment. Following $16 \mathrm{~h}$ of incubation, cells were fixed with methanol and stained with crystal violet. The number of migrated cells was counted in five randomly selected fields. Each experiment was repeated in triplicate.

Enzyme-linked immunosorbent assay (ELISA). HUVECs were seeded in 6 well plates at a density of $1 \times 10^{6}$ cells/well and were treated with Dra and LG or HG for $48 \mathrm{~h}$. The levels of VEGF in the supernatants were measured using an ELISA kit (R\&D Systems, Inc., Minneapolis, MN, USA) according to the manufacturer's instructions. The absorbance values were read within 30 min using an automatic microplate spectrophotometer (Anthos Zenyth 340st; Anthos Labtec Instruments GmbH, Salzburg, Austria) at $450 \mathrm{~nm}$ (A450). The average A450 values were calculated for each set of reference standards, controls and samples. A standard curve was constructed by plotting the mean absorbance obtained for each reference standard against its concentration using Excel software (Office 2010; Microsoft Corporation, Redmond, WA, USA). The concentration of each sample was then determined using the standard curve.

Reverse transcription-quantitative polymerase chain reaction (RT-qPCR). Total RNA was extracted using TRIzol Reagent (GenStar Biosolutions, Beijing, China), and the concentration of RNA was determined by absorbance at 260 and $280 \mathrm{~nm}$. A total of $2 \mathrm{mg}$ total RNA was reverse transcribed to cDNA using a PrimeScript ${ }^{\mathrm{TM}}$ RT reagent kit with gDNA Eraser (Takara Bio, Inc., Otsu, Japan). qPCR was conducted to measure mitogen-activated protein kinase 6 (MAPK6), RAS p21 protein activator 1 (RASA1) and VEGF gene expression. The $20 \mu \mathrm{l}$ reaction mixture contained $7.4 \mu \mathrm{l}$ nuclease-free water, $2 \mu \mathrm{l}$ cDNA, $0.1 \mu \mathrm{l}(10 \mu \mathrm{M})$ each primer, $0.4 \mu \mathrm{l}$ ROX Reference Dye (50X) and $10.0 \mu \mathrm{l} \mathrm{SYBR}{ }^{\circledR}$ Premix Ex Taq (Takara Bio, Inc.). The thermal cycling conditions for PCR was as follows: $95^{\circ} \mathrm{C}$ for $30 \mathrm{sec}, 40$ cycles of $95^{\circ} \mathrm{C}$ for $15 \mathrm{sec}$ and $60^{\circ} \mathrm{C}$ for $34 \mathrm{sec}$. PCR amplification was conducted using specific primers for VEGF (forward, 5'-ACG ATC GAT ACA GAA ACC ACG-3', and reverse, 5'-CTC TGC GCA GAG TCT CCT CT-3'), RASA1 (forward, 5'-GGG AGG CCG GTA TTA TAA CAG-3', and reverse, 5'-CCA ACG TTT TCC TTT GCC C-3') and MAPK6 (forward, 5'-GAA TGG CAA ATC TGG CTC AAT T-3', and reverse, 5'-ACA GTC CTC CCC ACC ACT CA-3'). Actin primers (forward, 5'-TTG CGT TAC ACC CTT TCT TG-3', and reverse, 5'-TCA CCT TCA CCG TTC CAG TT-3') were used as an internal control. The PCR products were measured using and ABI 7500 Fast Real-Time PCR Detection System (Applied Biosystems, Thermo Fisher Scientific, Inc.). The $2^{-\Delta \Delta C q}$ method was used to analyze the relative expression of each gene.

Western blotting. Following culture with Dra and LG or HG, total protein was extracted using a total protein extraction kit (Qiagen, Inc., Valencia, CA, USA) and the concentration was measured using a bicinchoninic acid protein assay kit (Beijing Transgen Co., Ltd., Beijing, China). Samples (10 $\mu \mathrm{l})$ were loaded onto $10 \%$ sodium dodecyl sulfate polyacrylamide gel electrophoresis. Subsequently the proteins were transferred to a polyvinylidene fluoride membrane (EMD Millipore, Billerica, MA, USA) and blocked in $2.5 \%$ non-fat dried milk for $1 \mathrm{~h}$ at room temperature. Following this, the membrane was incubated 
with antibodies against VEGF (1:3,000; cat. no. ab36844; Abcam, Cambridge, UK), Ras (1:3,000; cat. no. ab52939; Abcam), MAPK6 (1:5,000; cat. no. ab53277; Abcam) and actin (1:6,000; cat. no. ab8226; Abcam) overnight at $4^{\circ} \mathrm{C}$. Following washing three times with Tris-buffered saline $0.5 \%$ Tween 20 (TBST), membranes were incubated with goat anti-rabbit or mouse peroxidase-conjugated secondary antibodies (1:5,000; cat. nos. A0545 and SAB3701132, respectively; Sigma-Aldrich, St. Louis, MO, USA). The membrane was washed three times with TBST, and the signals were detected by Fusion SOLO chemiluminescence system (Analis sa/nv, Namur, Belgium).

Statistical analysis. Statistical testing was performed using SPSS software, version 12.0 (SPSS, Inc., Chicago, IL, USA). Data are expressed as the mean \pm standard deviation of the mean of the indicated number of experiments. Statistical comparison between experimental group and control was performed using one-way analysis of variance and followed by Student-Newman-Keuls test to examine differences between groups or chi-square test (for percentage). $\mathrm{P}<0.05$ was considered to indicate a statistically significant difference.

\section{Results}

Effect of Dra on HUVEC viability. To investigate the mechanism by which Dra may promote wound healing, an MTT assay was conducted to measure the viability of HUVECs under different concentrations of Dra. As presented in Fig. 1, following culture with $5.5 \mathrm{mM}$ glucose concentration (LG), $5.5 \mathrm{mM}$ glucose concentration plus $7.5 \mu \mathrm{M}$ Dra (LG + Dra), $25 \mathrm{mM}$ glucose (HG) and $25 \mathrm{mM}$ glucose plus $7.5 \mu \mathrm{M}$ Dra $(\mathrm{HG}+\mathrm{Dra})$ for $48 \mathrm{~h}$, Dra increased the cell viability under LG and $\mathrm{HG}$ concentrations.

Dra facilitates HUVECs cell migration. Cell migration is suggested to serve a key role during wound repair. To investigate this, a Transwell assay was conducted using Dra and different glucose concentrations (Fig. 2A). This indicated an increased in the number of migrated cells following Dra treatment with both LG and HG concentrations. The cell numbers were calculated and are presented in Fig. 2B.

Effects of Dra on tube formation of HUVECs. Endothelial cell proliferation and migration are involved in angiogenesis which leads to subsequent vascular structure formation. Therefore, a tube formation assay was performed to evaluate the ability of Dra to facilitate endothelial cell differentiation during angiogenesis. As presented in Fig. 3A, when HUVECs were cultured on Matrigel in the absence of Dra, HG was observed to reduce tube formation. However, when incubated with Dra, more complex and branched tubular structures were formed.

The results indicate that Dra may induce tube formation in $\mathrm{HG}$ concentrations and reduce tube formation in $\mathrm{LG}$ concentrations (Fig. 3B). Further analysis revealed the effect of the Dra treatment on the tube diameter under LG and $\mathrm{HG}$ concentrations. As presented in Fig. 3C, compared with the HG concentration, LG concentration induced the formation of narrower and more filamentous tubes. However, increased branched tubular structure was formed following Dra treatment under both low and high glucose concentration.

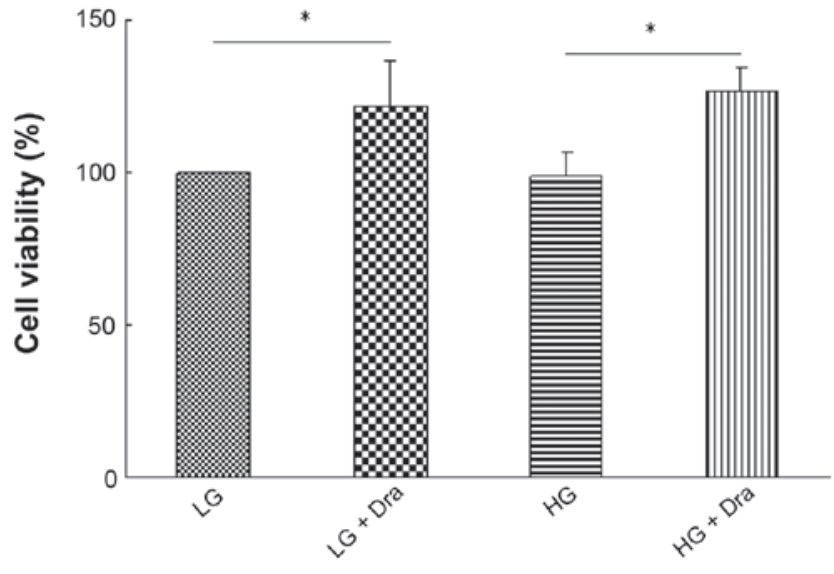

Figure 1. The effects of Dra on the viability of human umbilical vein endothelial cells. The proliferation effect of Dra was estimated by 3-(4,5-dimethylthiazol-2-yl)-2,5-diphenyltetrazolium bromide assay. The value from the baseline control group was set at $100 \%$. Data are presented as the mean \pm standard deviation from three individual experiments. ${ }^{*} \mathrm{P}<0.05$. Dra, dracorhodin perchlorate; LG, low glucose; HG, high glucose.

A
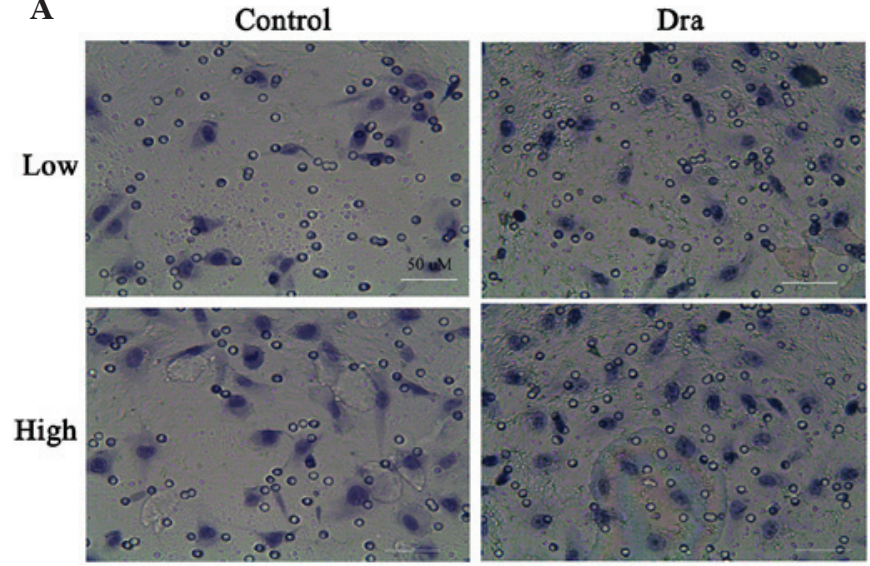

B

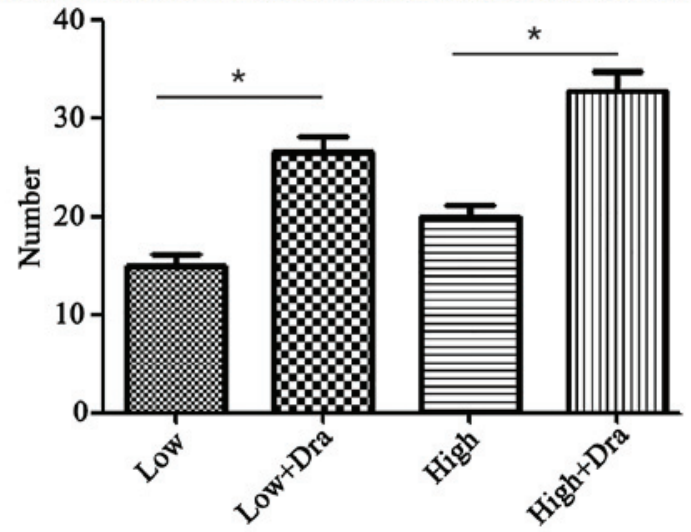

Figure 2. Effect of Dra on HUVEC migration. (A) Dra-induced HUVECs viability were photographed following staining with crystal violet. (B) Quantitative analysis of the Dra-induced HUVECs viability (MTT) were analyzed after the treatment with Dra and different concentrations of glucose. ${ }^{*} \mathrm{P}<0.05$, comparison indicated by brackets. Dra, dracorhodin perchlorate; HUVECs, human umbilical vein endothelial cells; MTT, 3-(4,5-dimethylthiazol-2-yl)-2,5-diphenyltetrazolium bromide.

Dra enhances the production of VEGF in HUVECs. In order to determine whether Dra treatment increased VEGF secretion by HUVECs, the VEGF levels were measured in the HUVECs using ELISA. HG treatment resulted in a reduction in VEGF 
A

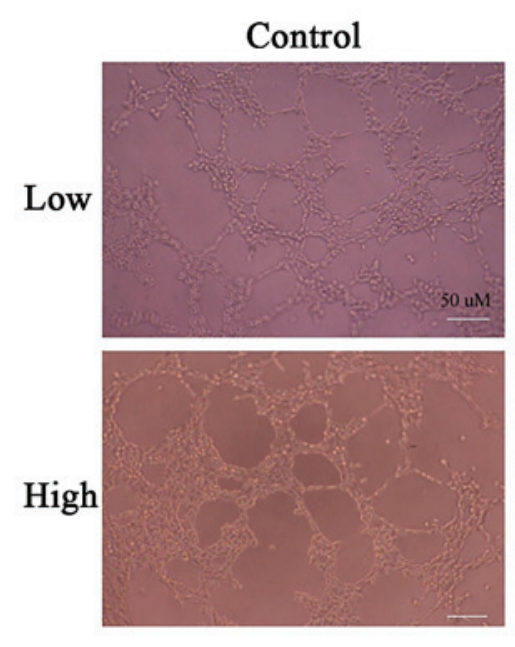

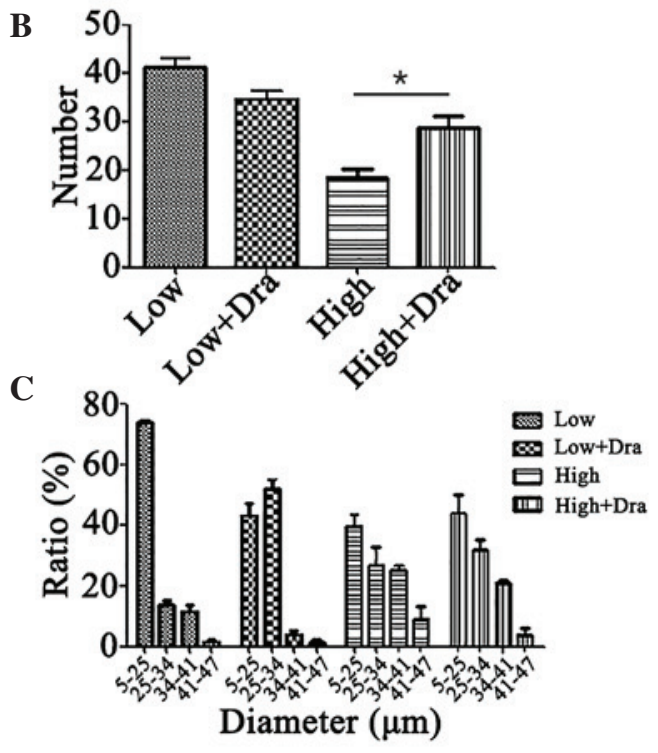

Figure 3. Effect of Dra on tube formation in HUVECs on matrigel. (A) Morphological alterations of HUVECs differentiating into vascular networks in three-dimensional matrigel following treatment with Dra for $6 \mathrm{~h}$. (B) Quantitative analysis of the total tube number in Dra-treated HUVECs using Image-Pro Plus software. (C) Distribution analysis of the total tube diameter in Dra-treated HUVECs using Image-Pro Plus software. Data are presented as the mean \pm standard deviation from four individual experiments. ${ }^{*} \mathrm{P}<0.05$, comparison indicated by brackets. Dra, dracorhodin perchlorate; HUVECs, human umbilical vein endothelial cells.

secretion (Fig. 4). Following Dra treatment, the secretion of VEGF was induced in both the LG and HG concentrations. Additionally, the ratio of VEGF increasing under low glucose concentration was significant compared with the ratio under high glucose concentration (Fig. 4).

MAPK signaling pathway analysis. The VEGF receptor (R)-Ras-MAPK signaling pathway serves an important role in the regulation of angiogenesis. Therefore, VEGF, Ras and MAPK protein expression levels were analyzed by western blotting (Fig. 5) and RT-qPCR (Fig. 6). The expression levels Ras protein and of VEGF, Ras and MAPK mRNA were suppressed by the HG concentration as shown in Figs. 5 and 6. These results were consistent with capillary growth suppression in diabetic ulcers (8). In addition, Dra was able to induce the expression levels of VEGF, Ras and MAPK under both LG and $\mathrm{HG}$ concentrations, with a greater effect observed in the LG concentration.

\section{Discussion}

Wound healing is a complicated process. There are at least three stages during the process of wound healing: Inflammation, proliferation and remodeling. The proliferation phase is characterized by granulation tissue formation, which primarily contains newly generated blood vessels, fibroblasts and collagen fibers. Endothelial cell and fibroblasts are considered to serve an essential role in the wound healing process in addition to angiogenesis. Angiogenesis during wound repair involves new blood vessel growth from endothelial cells, and serves a dual function of providing essential nutrients and oxygen to the wound site and promoting granulation tissue formation. However, in diabetic ulcer cases, healing impairment is caused by a number of physiological factors, with reduced angiogenesis being a main factor. Inhibition of

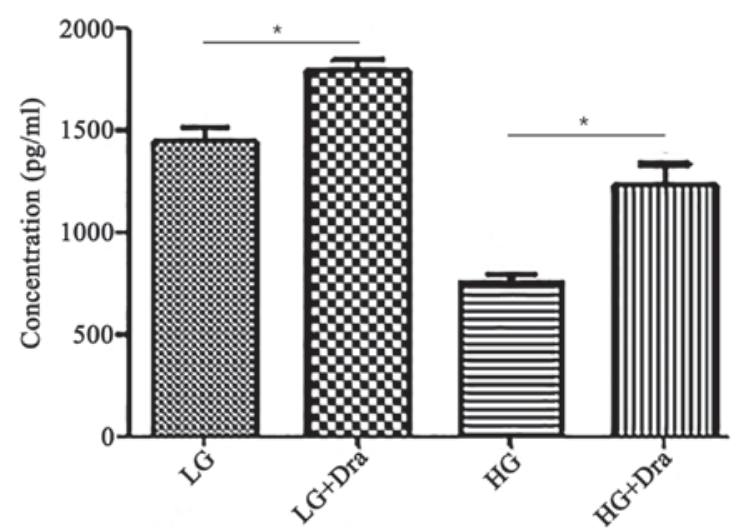

Figure 4. VEGF production in human umbilical vein endothelial cells Following Dra treatment, there was an increase in the production of VEGF both under LG and $\mathrm{HG}$ concentrations. ${ }^{*} \mathrm{P}<0.05$, comparison indicated by brackets. VEGF, vascular endothelial growth factor; Dra, dracorhodin perchlorate; LG, low glucose; HG, high glucose.

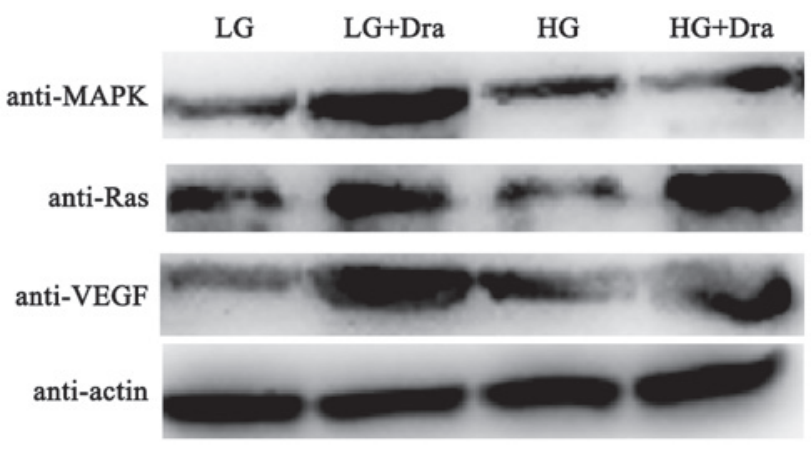

Figure 5. Western blot analysis of the expression of proteins in the VEGF signaling pathway induced by Dra. The expression levels of MAPK, Ras and VEGF are increased in HUVECs following treatment with Dra. Actin is used as a negative control. VEGF, vascular endothelial growth factor; Dra, dracorhodin perchlorate; MAPK, mitogen-activated protein kinase; HUVECs, human umbilical vein endothelial cells; LG, low glucose; HG, high glucose. 


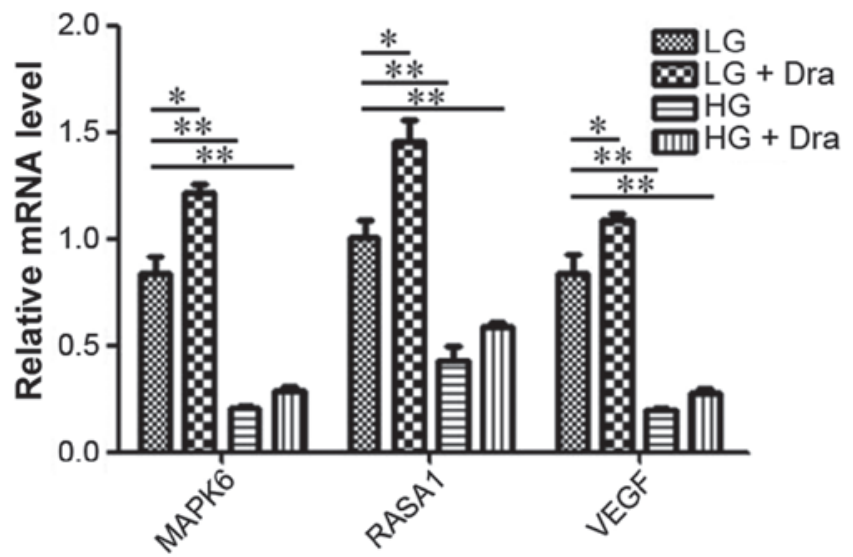

Figure 6. Measurement of mRNA expression levels of VEGF signaling pathway mediators by reverse transcription-quantitative polymerase chain reaction. The expression levels of MAPK6, RASA1 and VEGF are increased in HUVECs following treatment with Dra. Actin is used as a negative control. " $\mathrm{P}<0.05,{ }^{* *} \mathrm{P}<0.01$. VEGF, vascular endothelial growth factor; MAPK, mitogen-activated protein kinase; VEGF, vascular endothelial growth factor; HUVECs, human umbilical vein endothelial cells; Dra, dracorhodin perchlorate; LG, low glucose; HG, high glucose.

angiogenesis delays the wound healing process. Poor vasculature in diabetic wounds has been observed in diabetic mice, suggesting that dysfunction of the endothelium is a key factor in the pathogenesis of angiogenesis in diabetic ulcers (20). Therefore, agents that promote angiogenesis may improve diabetic wound healing.

Debridement, offloading and infection control are the main treatments for diabetic foot ulcers, and new treatments continue to be introduced. However, the majority of treatments are only effective for mild and moderate wounds, and the risk of amputation remains. Therefore, integration of traditional Chinese medicines with conventional treatments may aid in treating diabetic foot ulcers. Dragon's blood has long been used as a traditional Chinese medicine for improving blood circulation, inhibiting platelet aggregation and thrombus formation and healing wounds. Furthermore, dragon's blood has been indicated to have additional bioactivities, including alleviating insulin resistance, antimicrobial, anti-inflammatory and antioxidant effects $(17,21,22)$. Considering its predominant effect on circulation, the current study postulated that the effects of dragon's blood on chronic wounds induced by diabetes, may be attributed to the promotion of angiogenesis. Therefore, the effects of Dra, one of the main biological components of dragon's blood that can facilitate wound healing, were evaluated in angiogenesis, and the underlying mechanisms were explored.

Using an in vitro HG concentration in cultured HUVECs, it was possible to investigate the main steps of angiogenesis, in addition to the signaling pathway through which Dra may function. HUVECs are frequently used as in vitro model systems for various physiological and pathological processes, in particular in angiogenesis research. The proliferation, migration and formation of tubular structure by endothelial cells are indicators for the development of new blood vessels from the preexisting vascular bed in angiogenesis. The present study indicated that Dra was able to promote angiogenesis through increasing the proliferation, migration and tube formation of HUVECs, which are the initial stages of angiogenesis. The MTT assay indicated that Dra treatment was able to induce proliferation in HUVECs under both LG and HG concentrations. Furthermore, the migration of HUVECs was observed to be facilitated by Dra, in particular at the HG concentration, in addition to tube formation activity. These results are consistent with previous studies (23). However, further analysis revealed that Dra promoted the formation of thick tubes with large diameters under the different glucose concentrations. More highly branched tubular structures were observed to form following Dra treatment at both LB and HG concentrations.

It is notable that numerous types of cytokines and growth factors are responsible for inflammation, re-epithelialization and the formation of granulation tissue in the wound healing process. For example, VEGF is an important angiogenic cytokine, and promotes wound healing by stimulating proliferation and the migration of endothelial cells through the extracellular matrix. In addition, VEGF promotes the secretion of active growth factors and cytokines necessary for wound repair (23). In the present study, the production of VEGF by HUVECs was measured, and under both LG and HG, Dra was able to increase VEGF secretion.

VEGF activates various signaling pathways including phosphatidylinositol 3-kinase/Akt, protein kinase $\mathrm{C}$ and MAPK cascades. The Ras/MAPK signaling pathway is a signaling system that controls fundamental cellular processes including cell proliferation, differentiation, survival and migration (24). Receptor tyrosine kinases and their ligands often act as the inducers of this signaling system. The VEGFR/Ras/MAPK signaling pathways have been shown to be crucial downstream signaling targets involved in VEGF-induced angiogenesis, and both RAS and MAPK serve an important role in it. Therefore, the current study aimed to determine whether Dra treatment led to the activation of MAPK pathway by measuring Ras and MAPK expression levels. It was observed that in accordance with the VEGF expression, the mRNA expression levels of Ras and MAPK were downregulated in HG concentrations and upregulated by Dra treatment. In addition, the western blotting results indicated that VEGF, Ras and MAPK signaling were all activated by Dra treatment in HUVECs under HG conditions. From the results of the present study, it may be concluded that the effects of Dra on endothelial cells such as producing VEGF and activating the Ras/MAPK signaling pathway, are the cornerstones of Dra-mediated promotion of angiogenesis.

In conclusion, the current study indicates that promoting angiogenesis may be a potential mechanism for the therapeutic effects of dragon's blood on diabetic wounds. The function of Dra was observed to involve the activation of the Ras/MAPK signaling pathway in endothelial cells. The current study indicates that Dra, a traditional Chinese medicine isolated from dragon's blood, may hold potential as a clinical treatment for wound healing and blood vessel damage.

\section{References}

1. Bedell AJ: Retinal Vessel Proliferation in Diabetes. Trans Am Ophthalmol Soc 43: 271-276, 1945. 
2. Cholst MR, Levitt LM and Handelsman MB: Small vessel dysfunction in patients with diabetes mellitus. II. Retinal vessel response in diabetics following priscoline. Am J Med Sci 224: 39-41, 1952.

3. Colwell JA and Lopes-Virella MF: A review of the development of large-vessel disease in diabetes mellitus. Am J Med 85 (5A): 113-118, 1988.

4. Pedersen J and Olsen S: Small-vessel disease of the lower extremity in diabetes mellitus. On the pathogenesis of the foot-lesions in diabetics. Acta Med Scand 171: 551-559, 1962

5. Kavitha KV, Tiwari S, Purandare VB, Khedkar S, Bhosale SS and Unnikrishnan AG: Choice of wound care in diabetic foot ulcer: A practical approach. World J Diabetes 5: 546-556, 2014.

6. Falanga V: Wound healing and its impairment in the diabetic foot. Lancet 366: 1736-1743, 2005.

7. Walshe TE and D'Amore PA: The role of hypoxia in vascular injury and repair. Annu Rev Pathol 3: 615-643, 2008.

8. Cooper ME, Vranes D, Youssef S, Stacker SA, Cox AJ, Rizkalla B, Casley DJ, Bach LA, Kelly DJ and Gilbert RE: Increased renal expression of vascular endothelial growth factor (VEGF) and its receptor VEGFR-2 in experimental diabetes. Diabetes 48: 2229-2239, 1999.

9. Qaum T, Xu Q, Joussen AM, Clemens MW, Qin W, Miyamoto K, Hassessian H, Wiegand SJ, Rudge J, Yancopoulos GD and Adamis AP: VEGF-initiated blood-retinal barrier breakdown in early diabetes. Invest Ophthalmol Vis Sci 42: 2408-2413, 2001.

10. Advani A, Connelly KA, Advani SL, Thai K, Zhang Y, Kelly DJ and Gilbert RE: Role of the eNOS-NO system in regulating the antiproteinuric effects of VEGF receptor 2 inhibition in diabetes. BioMed Res Int 2013: 201475, 2013.

11. Chang Y, Chang TC, Lee JJ, Chang NC, Huang YK, Choy CS and Jayakumar T: Sanguis draconis, a dragon's blood resin, attenuates high glucose-induced oxidative stress and endothelial dysfunction in human umbilical vein endothelial cells. ScientificWorldJournal 2014: 423259, 2014.

12. Liu H, Lin S, Xiao D, Zheng X, Gu Y and Guo S: Evaluation of the Wound Healing Potential of Resina Draconis (Dracaena cochinchinensis) in Animal Models. Evid Based Complement Alternat Med 2013: 709865, 2013.

13. Zhang P, Li J, Tang X, Zhang J, Liang J and Zeng G: Dracorhodin perchlorate induces apoptosis in primary fibroblasts from human skin hypertrophic scars via participation of caspase-3. Eur J Pharmacol 728: 82-92, 2014.
14. Yu JH, Zheng GB, Liu CY, Zhang LY, Gao HM, Zhang YH, Dai CY, Huang L, Meng XY, Zhang WY and Yu XF: Dracorhodin perchlorate induced human breast cancer MCF-7 apoptosis through mitochondrial pathways. Int J Med Sci 10: 1149-1156, 2013.

15. Hu Q, Sun E, Xu FJ, Zhang ZH and Jia XB: Simultaneous content determination of notoginsenoside $\mathrm{R} 1$, ginsenoside $\mathrm{Rg} 1, \mathrm{Re}, \mathrm{Rb} 1$ and dracorhodin in ZJHX rubber paste by double wavelength HPLC. Zhongguo Zhong Yao Za Zhi 38: 2793-2797, 2013 (In Chinese).

16. Rasul A, Ding C, Li X, Khan M, Yi F, Ali M and Ma T: Dracorhodin perchlorate inhibits PI3K/Akt and NF- $\mathrm{B}$ activation, up-regulates the expression of $\mathrm{p} 53$, and enhances apoptosis. Apoptosis 17: 1104-1119, 2012.

17. He Y, Ju W, Hao H, Liu Q, Lv L and Zeng F: Dracorhodin perchlorate suppresses proliferation and induces apoptosis in human prostate cancer cell line PC-3. J Huazhong Univ Sci Technolog Med Sci 31: 215-219, 2011.

18. Xia MY, Wang MW, Cui Z, Tashiro SI, Onodera S, Minami M and Ikejima T: Dracorhodin perchlorate induces apoptosis in HL-60 cells. J Asian Nat Prod Res 8: 335-343, 2006.

19. Zhang P, Li J, Tang X, Zhang J, Liang J and Zeng G: Dracorhodin perchlorate induces apoptosis in primary fibroblasts from human skin hypertrophic scars via participation of caspase-3. Eur J Pharmacol 728: 82-92, 2014.

20. Roy DC, Mooney NA, Raeman CH, Dalecki D and Hocking DC: Fibronectin matrix mimetics promote full-thickness wound repair in diabetic mice. Tissue Eng Part A 19: 2517-2526, 2013.

21. Liu S, LiZ and Qian G: Quantitative determination of dracorhodin in Daemonorops draco B1. and traditional Chinese medicines containing Daemonorops draco B1. by HPLC. Hua Xi Yi Ke Da Xue Xue Bao 28: 450-453, 1997 (In Chinese).

22. Xia M, Wang D, Wang M, Tashiro S, Onodera S, Minami M and Ikejima T: Dracorhodin perchlorate induces apoptosis via activation of caspases and generation of reactive oxygen species. J Pharmacol Sci 95: 273-283, 2004.

23. Corral CJ, Siddiqui A, Wu L, Farrell CL, Lyons D and Mustoe TA: Vascular endothelial growth factor is more important than basic fibroblastic growth factor during ischemic wound healing. Arch Surg 134: 200-205, 1999.

24. Kolch W: Meaningful relationships: The regulation of the Ras/Raf/MEK/ERK pathway by protein interactions. Biochem J 351: 289-305, 2000. 